\title{
Novel Multichannel Plasma-Source Mass Spectrometer
}

\author{
Evan F. Cromwell \\ IBM Storage Systems Division, San Jose, California, USA \\ Peter Arrowsmith \\ Celestica, Inc., Toronto, Ontario M3C $1 \mathrm{~V} 7$, Canada
}

A novel mass spectrometer system for elemental analysis is described. The instrument combines an inductively coupled plasma (ICP) ion source with a Mattauch-Herzog mass spectrometer and multichannel ion detector. Ion detection is simultaneous and an elemental mass spectrum $(20-230 \mu)$ can be acquired in $<10 \mathrm{~ms}$. The instrument can be used with either Ar or He plasma sources. The speed of the system makes it well suited for acquisition of fast (10-100-ms duration) transient signals, such as those generated by pulsed laser ablation sample introduction. Preliminary system performance characteristics, which include detection limits, stability, and measurement accuracy, obtained with an Ar ICP are presented. The application of the instrument to the analysis of solid samples by laser ablation is discussed. (I Am Soc Mass Spectrom 1996, 7, 458-466)

$\mathrm{T}$ he technique of inductively coupled plasma mass spectrometry (ICP-MS) for elemental analysis is well known. [1-3] The mass spectrometer used in such instruments is generally a quadrupole filter or magnetic sector that is scanned sequentially to delect a single mass-to-charge $(\mathrm{m} / \mathrm{z})$ ratio at any one time. Instruments of this type are most suitable when there is a sufficient amount of analyte to produce a "steadystate $^{\prime \prime}$ signal by continuously flowing gases, nebulized solutions, liquid slurries, or ablated particulate material into the plasma. In practice, significant fluctuation of the ion signal is observed, especially on short time scales [4], and extensive signal averaging is required to obtain quantitative information. Analysis of transient signals, generated by introduction of discrete bursts of analyle into the plasma, is pussible with sequential instruments. However, when large numbers of isotopes and transients of duration $<1 \mathrm{~s}$ are analyzed, the duty factor per isotope and signal-to-noise ratio $(\mathrm{S} / \mathrm{N})$ suffer. If the amount of available sample is limited, it is necessary to have prior knowledge of which elements are present. Examples of sample introduction techniques that produce transient signals include flow injection [5-7] and direct injection nebulization [8] for solutions, and electrothermal vaporization $[9,10]$ and direct sample insertion for solids. [11, 12]. Other applications, such as single pulse laser ablation sampling and analysis of single particulates, tend to yield ion signals of shorter time duration. Many of the

Address reprint requests to Dr. Evan F. Cromwoll, Stormedia, Inc. 390 Reed Strect, Santa Clara, CA 95050. limitations to obtaining quantitative analysis from transient signals can be overcome by simultaneous ion detection.

Recently there have been a number of efforts to develop instruments with improved transient detection capabilities for elemental analysis. The combination of time-of-flight (TOF) [13] and quadrupole ion trap [14] mass spectrometers with an inductively coupled plasma (ICP) ion source have been demonstrated. TOF instruments are limited by low duty factor and space-charge in the extraction region, but may have advantages for the acquisition of very short $(<100 \mu \mathrm{s})$ transients. The ion trap has been shown to be more versatile, but suffers from dynamic range and mass calibration limitations. These mass spectrometers utilize single channel detecturs with sequential delection of ions. Instruments with true multichannel detectors have been developed. They include a magnetic sector instrument with multiple Faraday cup detectors to allow simultaneous detection of up to nine different isotopes [15] and a mass spectrometer equipped with an ion beam splitter and dual quadrupole filters to provide two independently tunable channels [16]. One advantage of these instruments is that correlated intensity fluctuations, for example, those caused by the arrival and volatilization of particles in the plasma, can be corrected by normalization to one of the signal channels, which leads to a significant increase in signal-to-noise ratio. Furthermore, the gain of each detector can be independently optimized to give the instrument a larger effective dynamic range. However, the number of isotopes that can be measured simultaneously is relatively small. 
One instrument designed for simultaneous detection of mass separated ions, but not previously used with an ICP, is the Mattauch-Herzog double-focusing mass spectrometer [17]. This spectrometer uses an electrostatic bending field and a magnetic field to image ions from an entrance slit onto a focal plane at the ex of the magnetic analyzer. The ions are focused, to first order, for angular and energy spreads, and separated along the focal plane by their mass-to-charge ratio. The design lends itself to small, compact instruments, well suited for the analytical laboratory [18]. With the development of sensitive microchannel plate (MCP) ion detectors, fast simultaneous detection of ions is possible. Combinations of a MCP detector with a Mattauch-Herzog mass spectrometer have been demonstrated previously [19-22].

We describe here the combination of a MattauchHerzog mass spectrometer and MCP detector with an ICP ion source, resulting in a versatile multichannel mass spectrometer (MCMS) for elemental analysis. The instrument has been run with both $\mathrm{Ar}$ and He ICP ion sources. Although the Ar ICP is a well characterized ion source, it presents challenges for use with a Mattauch-Herzog miass spectrometer because of the intense ${ }^{40} \mathrm{Ar}$ ion peak. Compared to an $\mathrm{Ar} \mathrm{ICP}$, the He ICP gives fewer mass spectral interferences and lower background signal. However, the He ICP is a less efficient ion source, and tends to form a secondary, or pinch, discharge between the ICP and the sampler nozzle. Results obtained with a He ICP will be published elsewhere. (Nam, S. H.; Montaser, A.; Cromwell, E. F., submitted for publication). The present article will discuss performance obtained with the Ar ICP. The new instrument has been used to analyze solution samples, as well as solid materials by laser ablation sample introduction [23, 24]. The absolute and relative performance is compared to a commercial instrument (ELAN 250, Perkin-Elmer, Norwalk, CT) that uses an Ar plasma and quadrupole mass spectrometer with a single channel electron multiplier (CEM) detector.

\section{Experimental}

\section{Vacuum System}

The various components of the ICP-MCMS are depicted in Figure 1. Four stages of differential pumping are used, which give an operating pressure of $\approx 1 \times$ $10^{-8}$ torr (Ar plasma) in the mass spectrometer chamber. The first stage is pumped by a mechanical pump whereas the other stages use turbomolecular pumps (TMPs). The system was designed with TMPs because of the need to pump He efficiently and minimize the background pressure of hydrocarbons. A summary of pump speeds, conductance limiting orifice dimensions, and chamber pressures is given in Table 1.

The plasma-vacuum interface is a water-cooled interface designed for an ELAN 5000 ICP-MS instrument (Perkin-Elmer). Standard sampler and skimmer cones

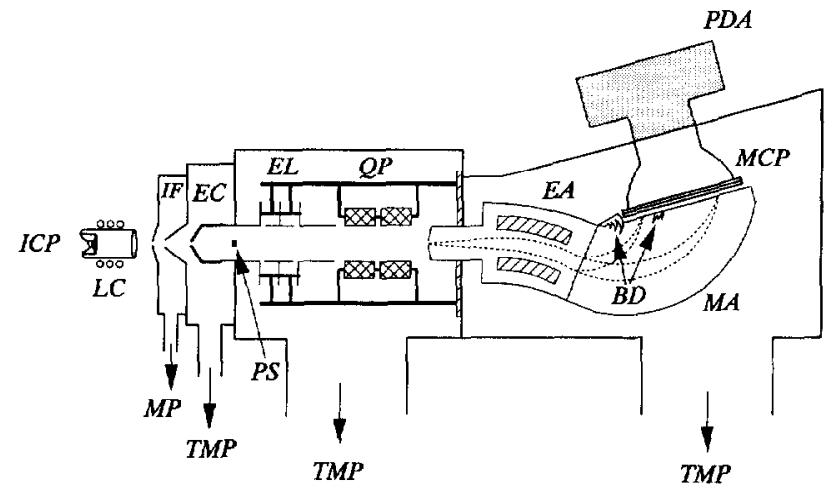

Figure 1. Schematic of the MCMS that shows the plasma torch, interface and differentially pumped vacuum chambers, ion optics, mass spectrometer, and detector (not to scale). Abbreviations: ICP, plasma torch (partial view); LC, load coil; IF, plasma interface chamber; EC, extractor cone; PS, photon stop/block; L1, lens 1; $\mathrm{QP}$, quadrupole lens pair; $\mathrm{EA}$, electric analyzer; $\mathrm{BD}$, ion beam dumps; $\mathrm{MA}$, magnetic analyzer; $\mathrm{MCP}$, microchannel plate and fiber optic bundle; PDA, photodiode array detector; MP, mechanical pump; TMP, turbomolecular pump.

for this interface are used. The conductance of the interface was increased by addition of a second 25mm-diameter pumping port. A pneumatically operated sliding plate valve is located downstream of the skimmer cone to allow isolation of the interface from the ion optic chambers. The remaining chambers were custom designed and built (Huntington Mechanical, Inc., Mountain View, CA), as was the vacuum pumping station (Leybold Vacuum Products, Inc, Export, PA).

\section{Plasma Source}

The instrument and impedance-matching network [26] were designed to enable switching between $\mathrm{Ar}$ and $\mathrm{He}$ ICPs with minimal change in configuration. Both use the same matching network and rf power supply, which give 5-kW maximum output at $40 \mathrm{MHz}$ (C5000, RF Plasma Products, Voorhees, NJ). Switching between the two ICPs requires the load coil, torch mount, and ground tap to be changed and different gas flows and capacitor settings. Operational parameters for the Ar ICP torch are given in Table 2. Operation with a He torch is discussed elsewhere (Nam, S. H.; Montaser, A.; Cromwell, E. F., submitted for publication). Solution samples were introduced by using an ultrasonic nebulizer with sample desolvation (U5000, Cetac, Omaha, NE). Solid samples were introduced by laser ablation by using the fourth harmonic of a Q-switched Nd:YAG laser $(266 \mathrm{~nm})$ and a custom built ablation chamber $[23,24]$.

\section{Ion Optics}

The mass spectrometer ion optics consisl of an extractor cone, a three-element electrostatic lens (lens 1), and a quadrupole lens pair (sec Figure 1). The ion optics and support structure are self-centered on the axis of 
Table 1. Parameters of the ICP-MCMS vacuum system

\begin{tabular}{|c|c|c|c|c|}
\hline & \multirow[b]{2}{*}{ Interface } & \multicolumn{2}{|c|}{ Vacuum chamber } & \multirow{2}{*}{$\begin{array}{c}\text { Mass } \\
\text { Spectrometry }\end{array}$} \\
\hline & & Second & Third & \\
\hline Main pump & $\begin{array}{l}\text { Leybold } \\
\text { SV180 }\end{array}$ & $\begin{array}{l}\text { Balzers } \\
\text { TMP500 }\end{array}$ & $\begin{array}{l}\text { Leybold } \\
\text { TMP1000 }\end{array}$ & $\begin{array}{l}\text { Leybold } \\
\text { TMP360V }\end{array}$ \\
\hline $\begin{array}{r}\text { Backing } \\
\text { pump }\end{array}$ & - & $\begin{array}{r}\text { Leybold } \\
\text { D40B }\end{array}$ & $\begin{array}{l}\text { Edwards } \\
\text { E2M18 }\end{array}$ & $\begin{array}{l}\text { Leybold } \\
\text { D16B }\end{array}$ \\
\hline $\begin{array}{l}\text { Pumping } \\
\text { speed }\end{array}$ & $127 \mathrm{ft}^{3} / \mathrm{min}$ & $500 \mathrm{~L} / \mathrm{s}$ & $1000 \mathrm{~L} / \mathrm{s}$ & $360 \mathrm{~L} / \mathrm{s}$ \\
\hline $\begin{array}{l}\text { Orifice } \\
\quad \text { diameter }(\mathrm{mm})\end{array}$ & 1.12 & 0.87 & 2.0 & $0.1 \times 4.5$ \\
\hline \multicolumn{5}{|c|}{ Chamber pressures (torr) } \\
\hline $\operatorname{Ar} I C P$ & 0.7 & $1 \times 10^{-3}$ & $6 \times 10^{-6}$ & $1 \times 10^{-8}$ \\
\hline Base & - & $<1 \times 10^{-7}$ & $<1 \times 10^{-7}$ & $5 \times 10^{-9}$ \\
\hline
\end{tabular}

Table 2. Plasma operating conditions

\begin{tabular}{ll}
\hline & Ar ICP \\
\hline \hline Gas flows (L/min) & \\
Plasma & 14.0 \\
Aux. & 2.0 \\
Sample & 1.0 \\
rf Power (kW) & $1.0-1.5$ \\
Load coil/ & $3 \frac{1}{2}$ Turns \\
grounding & center-tapped \\
torch & Sciex “long" type \\
Load coil-interface & 20 \\
distance (mm) & \\
\hline
\end{tabular}

the mass spectrometer and the axial positions of lens 1 and the quadrupole lens pair are independently adjustable. The extractor cone is mounted on and electrically connected to the first element of lens 1 and serves as the conductance-limiting orifice between the second and third chambers. The tip of the extractor cone is located close $(\approx 1 \mathrm{~mm})$ to the interface sliding plate valve. A negative potential is applied to the cone to increase the flux of ions through the center 2-mmdiameter hole. After extraction, the ions are transmitted through a short field-free region before they enter lens 1. A small photon stop ( $3 \mathrm{~mm}$ diameter), which is in electrical contact with the extraction cone, is located in this field-free region. A photon stop was necessary because, even though there is no direct line of sight between the plasma and the detector, significant amounts of scattered plasma photons reach the MCP and increase the background. This increased background is most noticeable for the high mass end of the detector, which is less shadowed by the mass spectrometer. Under the tuning conditions used, the photon stop caused an $\approx 20 \%$ decrease in signal; possible reasons for this small decrease are discussed in subsequent text. Lens 1 has an inner diameter of $25 \mathrm{~mm}$ and a center lens element length of $25 \mathrm{~mm}$. After lens 1, a quadrupole lens pair is used to focus the ion beam and improve the spatial overlap of the ion beam with the rectangular entrance slit of the mass spectrometer [26, 27]. The quadrupole elements are made from goldcoated aluminum and each has a diameter of $32 \mathrm{~mm}$ and length of $25 \mathrm{~mm}$. The two sets are spaced $6.4 \mathrm{~mm}$ apart. The bias voltage of the quadrupoles is set at the float voltage of the mass spectrometer. Shields at this same potential are located before and after the quadrupole lens to reduce fringe fields. The potentials of the second quadrupole lens elements are independently adjustable to facilitate alignment of the ion beam with the entrance slit of the mass spectrometer. Typical potentials applied to the various elements are given in Table 3.

\section{Mass Spectrometer}

The mass spectrometer is of the Mattauch-Herzog [17] geometry with a 95-mm-long focal plane. The unit was custom built and is similar in design to mass spectrometers developed at the University of Minnesota [19]. The spectrometer is housed inside the final vacuum chamber; the mass spectrometer entrance slit (width $100 \mu \mathrm{m}$ and height $4.6 \mathrm{~mm}$ ) serves as a conductance-limiting orifice to that chamber. A secondary $(\alpha)$ slit of dimensions $2 \mathrm{~mm} \times 3 \mathrm{~mm}$ is mounted before the electric sector to improve angular focusing. This slit limits the angular divergence of the beam to $2^{\circ}$. Both slits are detachable to allow various slit widths to be used. The center of the electric sector has a radius of $107 \mathrm{~mm}$, and the spacing between the electric sectors is $10 \mathrm{~mm}$. The inside of the electric sector is coated with

Table 3. Ion optic potentials

\begin{tabular}{|c|c|c|}
\hline \multicolumn{2}{|l|}{ Element } & Potential (V) \\
\hline \multicolumn{2}{|l|}{ Interface } & 0 \\
\hline \multicolumn{2}{|l|}{ Extractor cone } & -500 \\
\hline \multicolumn{3}{|l|}{ First ion lens } \\
\hline \multicolumn{2}{|l|}{ First } & -500 \\
\hline \multicolumn{2}{|l|}{ Second } & -1800 \\
\hline \multicolumn{2}{|l|}{ Third } & -400 \\
\hline \multicolumn{3}{|c|}{ Quadrupole lens pair } \\
\hline \multirow[t]{2}{*}{ First lens } & Horizontal & -440 \\
\hline & Vertical & -360 \\
\hline \multirow[t]{2}{*}{ Second lens } & Horizontal & -370 \\
\hline & Vertical & -430 \\
\hline \multicolumn{3}{|c|}{ Mass Spectrometer } \\
\hline \multicolumn{2}{|c|}{ Float } & -400 \\
\hline \multicolumn{2}{|c|}{ Positive elestrode } & -353 \\
\hline \multicolumn{2}{|c|}{ Negative electrode } & -447 \\
\hline
\end{tabular}


graphite (Aerodag G, Acheson, Akron, $\mathrm{OH}$ ) to attenuate scattered plasma light. The active focal plane (i.e., where the detector is located) of the magnetic sector extends from a radius of 18 to $72 \mathrm{~mm}$ and the magnetic sector gap is $4.6 \mathrm{~mm}$. The magnet poles are made from Alnico 9 and the yoke and pole faces are ingot iron. The magnetic field strength of $5500 \mathrm{G}$ at the exit of the magnetic analyzer is uniform across the detector plane. An ion collector located at the low mass end of the exit of the magnetic sector serves to trap the intense He ion beam produced by the He ICP. For the Ar ICP, the detector is shielded over $a \approx 10-\mu$ region centered around $40 \mu$ by a small ion beam dump positioned close to the focus of the Ar ions.

The mass spectrometer is mounted on the detector assembly (with a 2-mm gap between the magnetic sector and the MCP) and positioned such that the design ion focal plane coincides with the front of the ion detector. This setup necessarily means that the ion focus is located outside the region of uniform magnetic field. Aberrations caused by this arrangement can be compensated by accelerating the ions onto the detector face. In practice, the optimum focus is obtained by tuning the electric sector polentials and the detector electron deflector shield potential. Mu-metal shields located at the exit of the magnetic sector reduce the effect of magnetic fringe fields on electron trajectories in the MCP detector [28].

\section{Ion Detector}

The ion deteclor is a modified Chevron dual microchannel plate electron multiplier coupled to a photodiode array (PDA) detector (Galilco Electro-Optics, Sturbridge, MA). The MCP was designed to match the focal plane of the mass spectrometer and has dimensions $76 \mathrm{~mm} \times 6.4 \mathrm{~mm}$. The gain of the dual MCP is $\approx 1 \times 10^{6}$ at a voltage of $2.0 \mathrm{kV}$ across the plates. An electron deflector shield is located between the front of the MCP and the mass spectrometer to reduce background signal caused by scattered electrons. The output of the MCP is accelerated onto a phosphor screen and the resulting fluorescence is coupled via a fiber optic bundle (length $102 \mathrm{~mm}$ with 3:2 image reduction) onto a 51-mm-long PDA with 2048 elements spaced 25 $\mu \mathrm{m}$ apart. The fiber optic bundle also serves as a vacuum feedthrough to the PDA, which is located outside the vacuum chamber. The PDA is cooled to $-30{ }^{\circ} \mathrm{C}$ to reduce dark signal. The PDA is operated by a 16-bit controller and software (ST-1000, Princeton Instruments, Trenton, $\mathrm{NJ}$ ) and data are collected and analyzed by $\mathrm{PC}$. Operating parameters for the detector are listed in Table 4.

\section{Data Acquisition and Analysis}

The PDA signals are recorded in analog mode because of the high ion flux on the detector. Under the condi-
Table 4. Detector operational parameters

\begin{tabular}{lc}
\hline Parameter & Value \\
\hline \hline $\mathrm{e}^{-}$Deflector shield & $-700 \mathrm{~V}$ \\
MCP Front plate & $-800 \mathrm{~V}$ \\
$\quad$ Back plate & $+800 \mathrm{~V}$ \\
Phosphor screen & $+4 \mathrm{kV}$ \\
Minimum readout time & $8 \mathrm{~ms}$ \\
Background counts & $<0.1 / \mathrm{s}$ \\
$\quad$ (plasma off) & $120 \mu \mathrm{m}$ \\
Detector resolution (FWHM) &
\end{tabular}

tions listed in Table 4, a single ion will produce a peak with an intensity of approximately 50-100 PDA counts. Signal intensities can be controled by variation of the signal acquisition time from $8 \mathrm{~ms}$ to a practical maximum of $500 \mathrm{~ms}$. The upper limit arises from saturation of a significant portion of the detector by plasma ion species $\left(\mathrm{N}, \mathrm{O},{ }^{36} \mathrm{Ar}, \mathrm{Ar}_{2}\right.$, etc.). Installation of beam dumps for these species might allow longer acquisition times. The maximum readout signal from the PDA is 65,000 , which affords up to 4 orders of magnitude dynamic range. The dynamic range can be extended by variation of the PDA integration time.

For analysis of steady-state signals, the data acquisition is triggered in "free-running" mode and spectra are acquired continuously. For analysis of transient signals generated by a single laser pulse, data acquisition is triggered by the laser and synchronized with the arrival of the ions at the detector by a pulse delay generator (DG535, Stanford Research Systems, Palo Alto, CA). The arrival time of the ion signal has a jitter of 100-200 ms caused by variation in the transport of ablated material between the ablation cell and the ICP. The detector is triggered $200 \mathrm{~ms}$ before the average signal arrival time to ensure complete acquisition of the signal.

A single spectrum can be acquired with integration time equal to the duration of the transient signal. Alternatively, multiple spectra, with shorter integration times can be acquired during a given transient, which thereby allows control over peak signal intensities and dynamic range. Data from a single laser event are acquired as $n$ consecutive PDA exposures of $m$ millisecond duration, such that $m * n=400 \mathrm{~ms}$. Individual mass spectra acquired with the same integration time are averaged together to improve the $\mathrm{S} / \mathrm{N}$ ratio. Peak areas and/or heights are used to calculate isotope intensities. For relatively well separated peaks, areas are obtained by integration of mass spectra. Because of the relatively poor mass resolution of the MCMS, it is necessary to perform deconvolution to determine the area and height of closely spaced peaks. To do this a pseudo-Voigt line shape is fitted to each peak by using a nonlinear least squares program (Origin Peak Fitting Module, MicroCal, Cambridge, MA). The pseudo-Voigt function is a linear combination of Gaussian and Lorentzian functions with variable weighting that allows a wide varicty of peak shapes to be fitted. 


\section{Results and Discussion}

\section{Performance Characteristics}

The performance of the multichannel mass spectrometer was characterized by using the Ar ICP to allow direct comparison with a commercial sequential ICP instrument (ELAN 250 with upgraded quadrupole mass filter and ion optics, Perkin-Elmer). A solution that contained five elements ( $\mathrm{Al}, \mathrm{Co}, \mathrm{Y}, \mathrm{Rh}$, and Ho, designated solution $\mathrm{A}$ ) was used to measure instrument linearity, detection limits, and stability. Monoisotopic elements were chosen for ease of data analysis. A typical mass spectrum of solution $A$ at $50-\mathrm{ng} / \mathrm{g}$ concentration is shown in Figure 2.

Linearity of Instrument Response. Five dilutions of a $5-\mu \mathrm{g} / \mathrm{g}$ solution A ranging from $10 \mathrm{ng} / \mathrm{g}$ to $1 \mu \mathrm{g} / \mathrm{g}$ were used to assess linearity. Mass spectra of each of the six solutions were acquired with exposure times of 10,100 , and $500 \mathrm{~ms}$ on the MCMS. Nine spectra were averaged for each exposure time and the areas of the five analyte peaks were determined with background correction. The results for 100-ms exposures are shown in Figure 3a.

Good linearity is obtained between $50 \mathrm{ng} / \mathrm{g}$ and $1-\mu \mathrm{g} / \mathrm{g}$ concentration at $100-\mathrm{ms}$ exposure. The $5-\mu \mathrm{g} / \mathrm{g}$ solution slightly salurated the detector at this exposure, whereas the signals for the $10-\mathrm{ng} / \mathrm{g}$ solution exhibited larger variation possibly duc to poor counting statistics. At $10-\mathrm{ms}$ exposure, the low concentration spectra exhibited significant deviations from linearity, but the response for the $500-\mathrm{ng} / \mathrm{g}$ to $5-\mu \mathrm{g} / \mathrm{g}$ solutions was similar to the $100-\mathrm{ms}$ data. At 500 -ms exposure, good linearity was obtained between $10 \mathrm{ng} / \mathrm{g}$ and 500 $\mathrm{ng} / \mathrm{g}$, but higher concentrations saturated the detector. In general, good linearity is obtained for peak intensi-

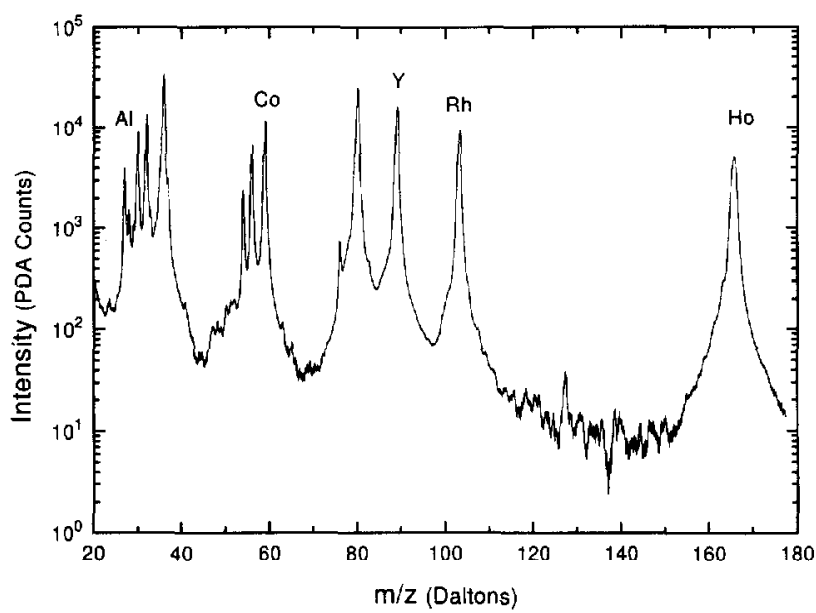

Figure 2. Mass spectrum acquired by the MCMS of a $50-\mathrm{ng} / \mathrm{g}$ multielement solution $A$ (see text). The spectrometer was at a potential of $-400 \mathrm{~V}$, which gave a mass range of 12-178 $\mu$, and the spectrum is the average of $27100-\mathrm{ms}$ exposures (only the $20-178 \mu$ region is shown).
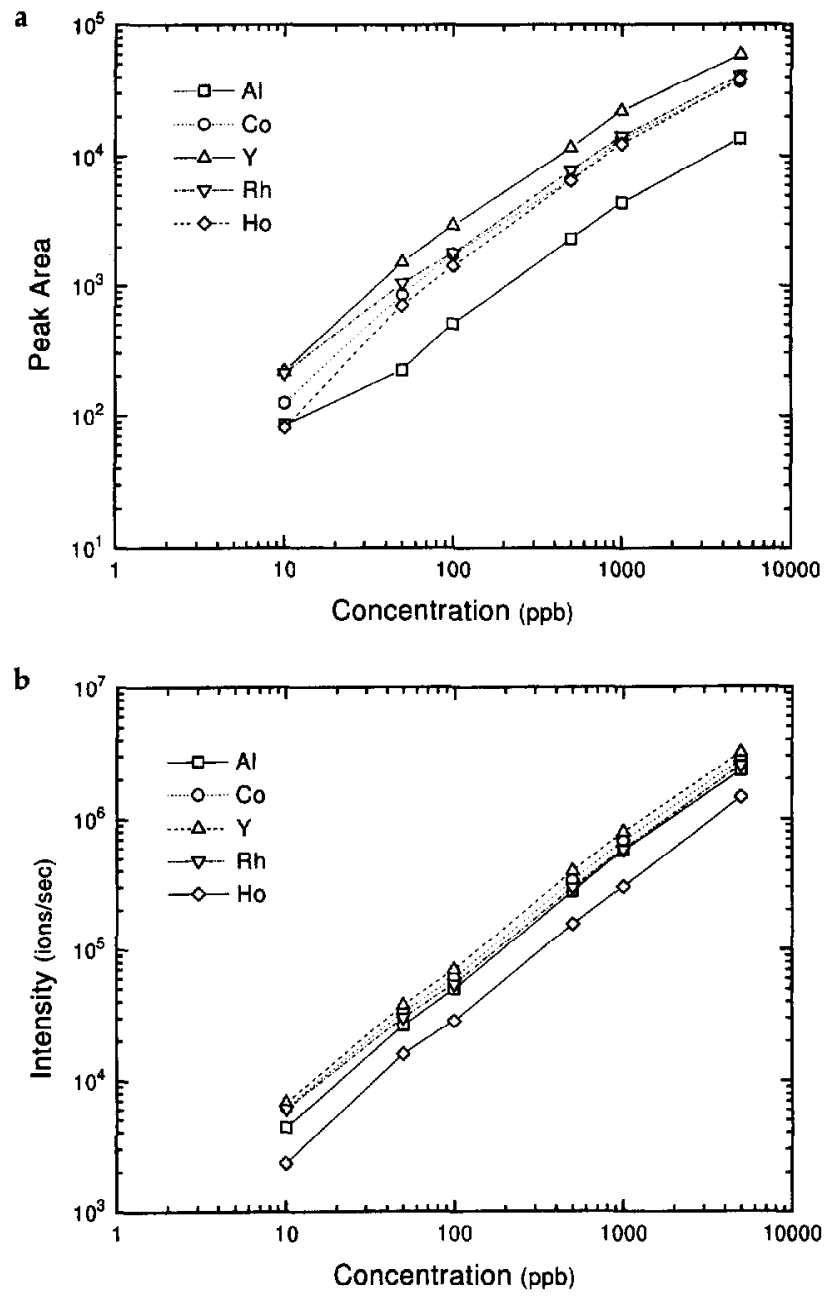

Figure 3. (a) Analytical curves measured on the MCMS for five analyte elements over the concentration range $10-5000 \mathrm{ng} / \mathrm{g}$. For each concentration, nine spectra acquired at $100-\mathrm{ms}$ exposure were averaged together. (b) Analytical curves measured on the ELAN 250 ICP-MS for the elements in a by using a peak-hopping mode of operation. For each concentration 200 repeats with 100-ms dwell time were averaged together.

ties up to $\approx 3 \times 10^{4}$ counts (which correspond to $50 \%$ of the saturation limit) for the range of exposure times studied.

The same solutions were analyzed with the ELAN quadrupole ICP instrument. The sample gas flow through the ultrasonic nebulizer was reduced to prevent detector saturation with the $5-\mu \mathrm{g} / \mathrm{g}$ solution. Signals were acquired in "peak-hopping" mode with a dwell time of $100 \mathrm{~ms}$ and averaged over 200 repeats. The results for the six solutions are shown in Figure $3 \mathrm{~b}$. Linear fits to the data in Figure $3 \mathrm{~b}$ give an average correlation coefficient $\left(R^{2}\right)$ of 0.9983 . Corresponding values for Figure $3 \mathrm{a}$ are 0.9885 for all data points and 0.9988 without the highest $(5 \mu \mathrm{g} / \mathrm{g})$ value. The total data integration time for the ELAN was $20 \mathrm{~s}$, compared to $0.9 \mathrm{~s}$ for the MCMS. The better linearity of the ELAN is believed to arise from improved counting statistics due to longer integration times and superior 
dynamic range of the CEM detector operated in pulsecounting mode compared to the MCP-PDA operated in analog mode. The latter limitation can be partly overcome by varying the exposure time of the PDA.

Detection Limits: Detection limits were calculated for the five analytes of solution A. The detection limit is defined as that concentration that gives a signal equivalent to $3 \times$ the standard deviation of the background signal at the mass-to-charge region of interest. Detection limits were obtained by linear extrapolation of the signal level that corresponds to the peak intensity obtained with a $50-\mathrm{ng} / \mathrm{g}$ solution. Background signal levels were determined from a blank spectrum acquired with a total integration time of $8.1 \mathrm{~s}$. Background signal arises from several sources. With the ion optics shut off, the background from dark current and plasma photons is typically $<1$ count per second per channel across the mass range. Note that without a photon block, this noise increases to a few hundred count per second in the high mass region. Under normal operation with the Ar ICP, the region up to $100 \mu$ exhibits significantly higher background and noise that is caused by the unresolved tails of intense plasma ion peaks. For example, with a 100 -ms exposure time the average standard deviation of the background for a PDA channel is 21 counts in the $60-70-\mu$ region, 13 counts in the $90-100-\mu$ region, and 5.5 counts above $130 \mu$. The background noise is close to statistical. With the preceding method, the calculated detection limits are Al: $0.4 \mathrm{ng} / \mathrm{g}, \mathrm{Co:} 0.1 \mathrm{ng} / \mathrm{g}, \mathrm{Y}: 0.04$ $\mathrm{ng} / \mathrm{g}$, Rh: $0.07 \mathrm{ng} / \mathrm{g}$, and Ho: $0.02 \mathrm{ng} / \mathrm{g}$. For continuous sample introduction, the sensitivity of the ICPMCMS is $1-2$ orders of magnitude poorer than that of the ELAN 250 under comparable operating conditions. These results are preliminary and are expected to improve as factors that affect instrument resolution (see following text) and beam transmission are better understood.

The present instrument was designed to achieve $100 \%$ detection duty cycle with transient ion signals, rather than high sensitivity with steady-state signals. To compare sensitivities for transient signals, the duty factor per isotope also should be considered. The duty factor of a quadrupole or other sequentially scanning instrument decreases with the number of isotopes to be monitored, or as the mass range increases. In the worst case, which corresponds to a large number of isotopes or detection over the full mass range, the duty factor is $\sim 10^{-2}$. There is an additional reduction in duty cycle that arises from the delay required to establish instrument stability following a mass change. This can be significant (1-10 ms per isotope) for peakhopping operation, and becomes noticeable when the total time exceeds the measurement time. The MCMS has two further advantages over single channel sequential instruments. The $\mathrm{S} / \mathrm{N}$ ratio can be improved by correcting the ion signal for fluctuations that are temporally correlated between two or more isotopes, for example, signal fluctuations produced by sample introduction of droplets or particulates. Second, because a complete mass spectrum is recorded, no prior knowledge of the elements present in an unknown sample is required. A detailed comparison of the $S / N$ between the MCMS and the quadrupole mass spectrometer for transient signal acquisition will be the subject of future experiments.

The sensitivity and resolution of the MCMS instrument are believed to be limited by the kinetic energy spread of the ions extracted from the plasma. This spread may arise from space-charge effects in the regions close to the sampler and skimmer nozzles or from the extraction of ions from the interface. The Mattauch-Herzog mass spectrometer compensates for ion kinetic energy spread to the first order. However, the resolution is degraded if the spread in kinetic energy $(\Delta E)$ is comparable to the total kinetic energy of the ions in the mass analyzer region. The total kinetic energy (KE) is the sum of the initial ion KE and the acceleration potential from the interface to the mass spectrometer (i.e., the mass spectrometer float voltage).

In the present MCMS instrument, the resolution becomes too poor for practical purposes when the ion optics are tuned to give the maximum ion signal. Useful mass resolution is obtained at $10-100 \times$ reduced signal intensity by sampling a less intense portion of the ion beam, presumably with a lower kinetic energy spread. Under the current tuning conditions, unit mass resolution is achieved out to $\approx 130 \mu$. It is important to note that although peak spatial widths (measured in PDA pixels) are fairly constant across the mass range, peak separation decreases as $1 /(\mathrm{m} / \mathrm{z})$ which causes resolution to degrade at high mass. This degradation is the reason for the large tailing of the Ho peak in Figure 2, compared to the lower mass analytes. For this type of magnetic sector instrument $\Delta E / E$ may be reduced, and the sensitivity improved, by increasing the float voltage of the mass analyzer. However, in practice the permanent magnetic field of the mass spectrometer limits the maximum acceleration voltage that can be used. The possibility of increasing the magnetic field to allow higher acceleration voltages is being explored. Another possibility that is being explored is to limit the spread of ion kinetic energies that enter the magnetic sector with a KE-limiting slit; however, the slit will attenuate the ion flux to some extent.

Stability. The relative stability of the ion signal of the MCMS was characterized by taking spectra of solution A $(50 \mathrm{ng} / \mathrm{g})$ at various times over a $3-\mathrm{h}$ period. The instrument was warmed-up (plasma on, vacuum interlock open) for $\approx 1 \mathrm{~h}$ and the ion optics were tuned prior to data acquisition. A total of 6 spectra were taken over $3 \mathrm{~h}$; each comprised the average of 27 $100-\mathrm{ms}$ exposures. The intensities of the five analytes were determined from the peak area and $Y$ was used as the internal standard (IS). The relative intensities for 
each spectrum as a function of time are shown in Figure 4 . The average intensity and standard deviation of the analytes are Al: $0.143 \pm 0.011, \mathrm{Co}: 0.555 \pm 0.021$, $\mathrm{Rh}: 0.617 \pm 0.013$, and $\mathrm{Ho}: 0.529 \pm 0.033$. The average relative standard deviation (rsd) is $\pm 5 \%$. The absolute drift was worse, with rsd of $13 \%$ over $3 \mathrm{~h}$.

The drift in the relative intensities does nol appear to be systematic or to correlate with mass. The magnitude of the noise is similar to that associated with ICP signal fluctuations (see isotope abundance measurements which follow) and the drift in relative ion intensity is comparable to a commercial quadrupole instrument. Thus, under steady-state conditions, after running for a period, the stability of the MCMS appears acceptable.

Relative sensitivity factors (RSFs, with IS $=Y$ ) for the five elements of solution $\mathrm{A}$ were calculated and compared to RSFs obtained with the quadrupole ICPMS at the same plasma generator power $(1.3 \mathrm{~kW})$. These values are given in lable 5. 'I he MCMS has reduced response at low mass-to-charge ratio $(<60$ $\mu$ ). This is probably caused by the tuning of the ion optics and mass spectrometer, which are optimized for higher mass elements. Based on the small number of elements used, the response at higher mass appears to be more uniform than that of the ELAN 250 instrument.

\section{Transient Signal Analysis}

When pulsed laser sampling is used for bulk analysis of solids, a "steady-state" ICP-MS signal is generated by ensuring the duration of the transient signal that arises from the ablated material is greater than the interval between laser pulses. The transient can be lengthened by increase of the volume of the ablation

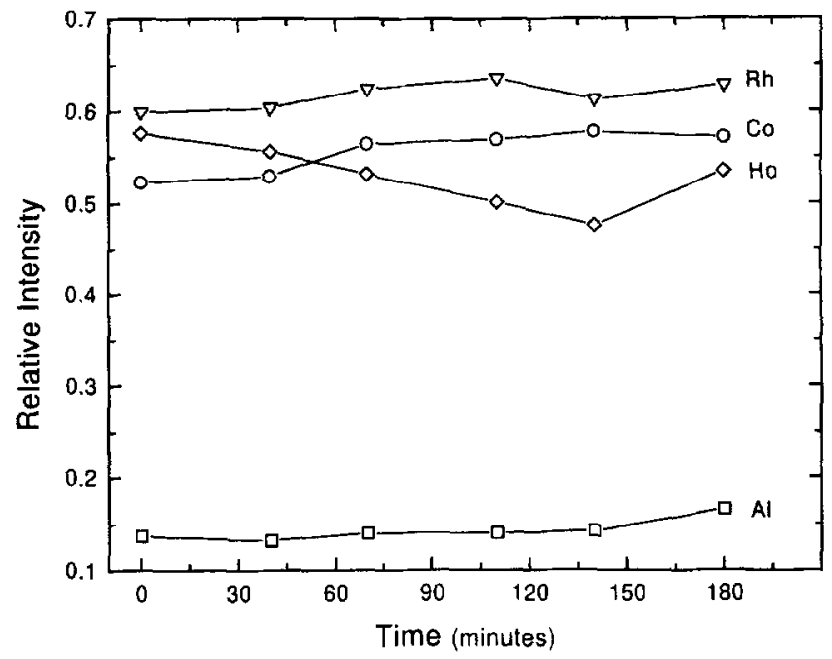

Figure 4. Relative stability of ion signals obtained with the MCMS for a 50-ng/g multielement solution A (see text) over a 3-h time period. Yttrium (not shown) is used as the internal standard with a value of 1 . At each time measurement, nine spectra acquired at a 100-ms exposure were averaged together.
Table 5. RSF comparison between MCMS and ELAN 250

\begin{tabular}{lccccc}
\hline & $\mathrm{Al}$ & $\mathrm{Co}$ & $\mathrm{Y}$ & $\mathrm{Rh}$ & $\mathrm{Ho}$ \\
\hline $\mathrm{MCMS}$ & 0.043 & 0.368 & 1.00 & 0.714 & 0.981 \\
ELAN 250 & 0.148 & 0.375 & 1.00 & 0.877 & 1.41
\end{tabular}

cell, reduction of the gas flow rate, or exterision of the transfer tube between the cell and the ICP. Although this makes the signal compatible with sequential mass spectrometers, the peak signal intensity and overall sensitivity are decreased. With a simultaneous instrument, such as the MCMS, the duration of the ablation signal can be matched to the relatively short data accumulation time, to give higher peak signal intensity and improved $\mathrm{S} / \mathrm{N}$ ratio. The temporal response of the ablation cell used in the present work (measured with the quadrupole instrument) is shown in Figure 5. This "high-fluence" cell, used for bulk analysis of materials [29], has a signal duration of $\approx 200 \mathrm{~ms}$ and produces a steady continuous signal with a $10-\mathrm{Hz}$ repetition rate laser.

To produce shorter duration signals, a second ablation cell has been developed. The temporal response of this cell also is shown in Figure 5. This cell has $10 \%$ of the volume of the high-fluence cell and is intended for ablation of small $(<10-\mu \mathrm{m})$ arcas, such as inclusions, surface contaminants, or particulates, and for low resolution $(1-\mu \mathrm{m})$ depth profiling $[30,31]$. As expected, the transient ion signal obtained with the smaller ablation cell has shorter duration and higher peak intensity for an equivalent amount of ablated material.

The capabilities of the MCMS for analysis of transient signals was evaluated by laser ablation of a Permalloy standard reference material (SRM 1160, NIST). The larger volume ablation cell was used with a laser fluence of $1.5 \mathrm{~J} / \mathrm{cm}^{2}$. Reduction of the detector gain $(1.4 \mathrm{kV})$ was necessary to prevent saturation of

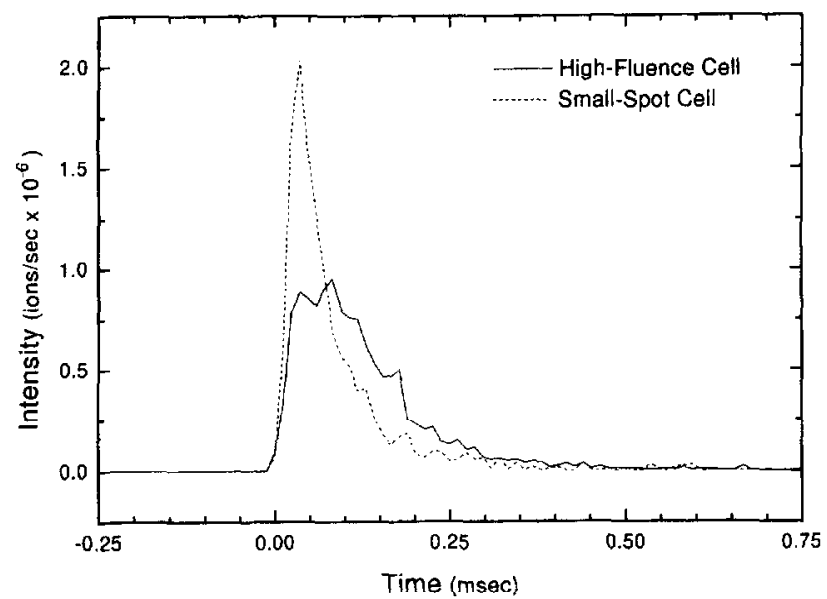

Figure 5. Comparison of the temporal response of the highfluence and the small-spot ablation cells. Each trace is the average of four measurements obtained with the ELAN 250 ICP-MS by using peak-hopping with a dwell time of $20 \mathrm{~ms}$. The peak areas are normalized. The signal shown is $\mathrm{Al}$, generated by ablation of aluminum alloy, SRM 1256A. 
the major element peaks. For this analysis, the laser was operated at $1-\mathrm{Hz}$ repetition rate and the laser beam was manually shuttered to regulate the number of pulses incident upon an area of the sample. Four spectra, each with 100-ms duration, were taken per laser pulse. The most intense spectra from each ablation event were averaged together to improve the $\mathrm{S} / \mathrm{N}$ ratio. A MCMS spectrum of the Permalloy sample obtained with seven laser pulses is shown in Figure 6. The main constituents of SRM $1160(80.3 \% \mathrm{Ni}, 14.3 \%$ $\mathrm{Fe}, 4.3 \% \mathrm{Mo}$ ) and their isotopes are all resolved. Small peaks at 52 and $65 \mu$, from $\mathrm{Cr}(0.05 \%)$ and $\mathrm{Cu}(0.021 \%)$, respectively, are present also. Ba (not certified in SRM 1160 ) is also observed. At the low fluence used, the amount of material ablated per pulse is $\sim 1 \mathrm{ng}$ [31]. From the estimated detection limit of $0.05 \%$ for a single laser pulse, the absolute detection limit of the MCMS is $\sim 0.5 \mathrm{pg}$. (For comparison, the mass of a $1-\mu \mathrm{m}$-diameter particle with density $5 \mathrm{~g} / \mathrm{cm}^{3}$ is $8 \mathrm{pg}$.) Use of the small-spot ablation chamber and a higher detector gain will improve the sensitivity further.

The accuracy of measurements made with MCMS can be estimated by comparison of isotope ratios for Mo. The region of the spectrum shown in Figure 6 from 90 to $102 \mu$ was fitted to seven pseudo-Voigt line shapes. The peaks in this region and the resulting fit are shown in Figure 7. The isotope ratios were calculated by dividing the area of each fitted peak by the total area of the seven isotopes. The measured and expected isotope ratios for Mo are given in Table 6 [32]. The average difference is $5.5 \%$. The accuracy is comparable to that expected with a quadrupole instrument with similar signal acquisition times, although it is 1-2 orders of magnitude worse than that obtainable with a multicollector instrument designed for isotope ratio work [15]. Causes of this deviation are expected

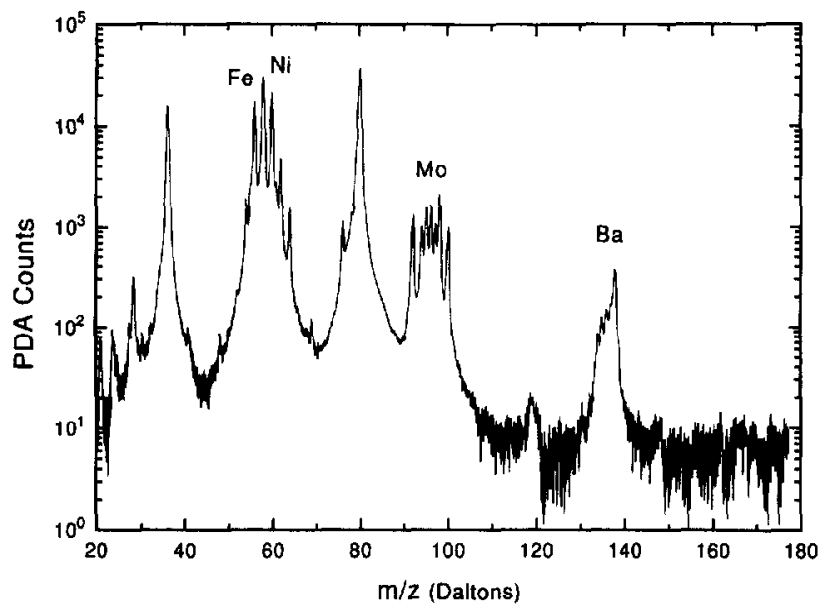

Figure 6. MCMS spectrum from laser ablation of Permalloy SRM $1160(80.3 \% \mathrm{Ni}, 14.3 \% \mathrm{Fe}, 4.3 \% \mathrm{Mo})$. The spectrum shown is the average of seven ablation events, with one 100-ms exposure per event. The spectrometer was at a potential of $-400 \mathrm{~V}$, which gave a mass range of $12-178 \mu$ (only the $20-178-\mu$ region is shown). Elements found in the sample are labeled.

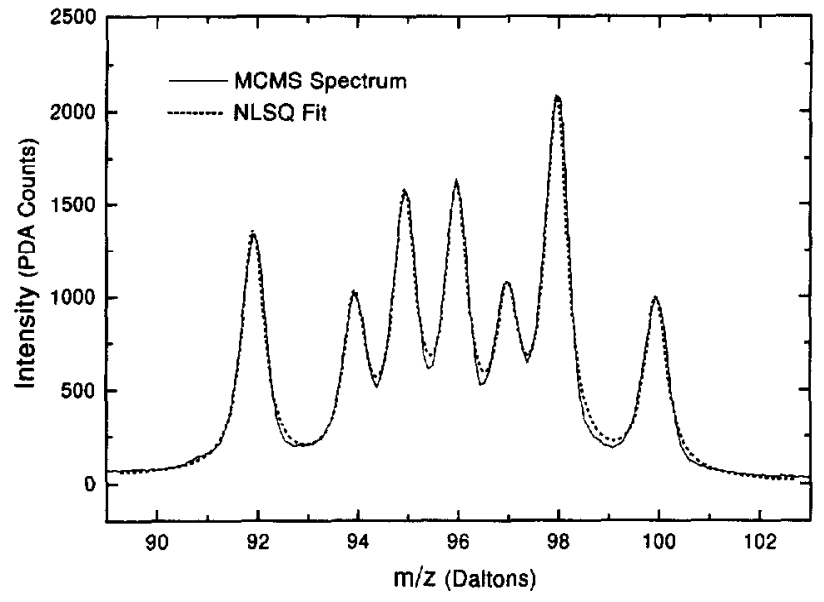

Figure 7. Expanded region of the mass spectrum of Figure 6 that shows the Mo isotopes. The nonlinear least squares fit of a pseudo-Voigt line shape to each peak is shown. The results of these fits are presented in Table 6 .

to include random effects, such as counting statistics (estimated to be $\approx 4 \%$ ) and plasma fluctuations, and systematic errors, which include peak-fitting error and isobaric interferences caused by isotopes of FeAr and NiAr.

\section{Conclusions}

A novel plasma-source multichannel mass spectrometer for simultaneous detection of ions over the elemental mass range has been developed. The design is optimized for detection and analysis of transient signals, especially those produced by laser ablation sample introduction. The instrument has poorer sensitivity, compared to commercially available sequential (quadrupole, sector-field) ICP-MS instruments, but the $100 \%$ duty factor of the system provides compensation, especially for acquisition of a full elemental mass spectrum. The MCMS also allows acquisition of a full mass spectrum in < $10 \mathrm{~ms}$ compared to $\approx 100 \mathrm{~ms}$ for a quadrupole and $\approx 600 \mathrm{~ms}$ for a rapid-scanning sector mass spectrometer. The stability of the instrument is comparable to a quadrupole instrument; the major source of instability is thought to be the vacuum interface, which has not been optimized yet. The major limitation is poor mass resolution, a consequence of

Table 6. Mo isotope ratio determination by ICP-MCMS (abundances given in percent)

\begin{tabular}{cccc}
\hline Isotope & MCMS & Expected & \% Difference \\
\hline \hline 92 & 15.0 & 15.84 & -5.37 \\
94 & 9.97 & 9.04 & +10.3 \\
95 & 16.0 & 15.72 & +1.72 \\
96 & 16.2 & 16.53 & -2.01 \\
97 & 9.37 & 9.46 & -0.94 \\
98 & 22.6 & 23.78 & -4.92 \\
100 & 10.9 & 9.63 & $+\frac{+12.9}{5.45 \%}$ \\
Average difference & & \\
\hline
\end{tabular}


the large spread in ion kinetic energy that occurs in plasma-source mass spectrometers. Compared to high resolution sector instruments $\left(m / \Delta m \leq 10^{4}\right)$, the resolving power of the MCMS is 2 orders of magnitude poorer. Further development, which includes increasing the ion acceleration potential and installation of energy-limiting slits, is underway to improve the resolution and enhance the performance of the system.

The capability of the MCMS to analyze transient signals has been demonstrated. It is possible to acquire a full mass spectrum with $100 \%$ duty factor from the 1-10 ng of material produced by a single laser pulse, with subpicogram detection limits. In contrast to sequential instruments, prior knowledge of elements of interest is not required for analysis of small amounts of unknown sample. This situation commonly arises in the analysis of thin films, particulates, surfaces contaminants, or inclusions. To fully realize the potential of the MCMS, the temporal width of the transient signal should be matched to the minimum spectrum acquisition time of $10 \mathrm{~ms}$.

\section{References}

1. Houk, R. S. Anal. Chem. 1986, 58, 97A.

2. Holland, G.; Eaton, A. N., Eds. Applications of Plasma Source Mass Spectrometry; Thomas Graham House: Science Park, Cambridge, UK, 1991.

3. Jarvis, K. W.; Gray, A. L.; Houk, R. S. Handbook of Inductively Coupled Plasma Mass Spectrometry; Blackie: Glasgow, Scotland, 1991.

4. Chan, W. T.; Russo R. E. Spectrochim. Acta 1991, 46B, 1471.

5. Beauchemin, D. Trenads Anal. Chem. 1991, 10, 71.

6. Hollenbach, M.; Grohs, I.; Mamich, S.; Kroft, M.; Denoyer, E. R. J. Anal. Atom. Spectrom. 1994, 9, 927.

7. Tyson, J. F.; Bysourth, S. R.; Brzeszczyk, E. A.; Debrah, E. Anal. Chem. Acta 1992, 261, 75

8. Wiederin, D. R.; Houk, R. S. Appl. Spectrosc. 1991, 45, 1408.

9. Gregoire, D. C.; Goltz, D. M.; Lamoureux, M. M.; Chakrabarti, C. L. I. Anal. Atom. Spectrom. 1994, 9, 919.
10. Carey, J. M.; Caruso, J. A. Crit. Rev. Anal. Chem. 1992, 23, 397.

11. Karanassios, B.; Horlick, G. Spectrochim. Acta Rev. 1990, 13, 89.

12. McLeod, C. W.; Routh, M. W.; Tikkanen, M. W. In Inductively Coupled Plasmas in Analytical Atomic Spectroscopy, 2nd ed.; Montaser, A.; Golightly, D. W., Eds.; VCH Publishers: New York, 1992; $\mathrm{p} 271$.

13. Myers, D. P.; Li, G.; Yang, P.; Heiftje, G. M. I. Am. Soc. Mass Spectrom. 1994, 5, 1008.

14. Koppenaal, D. W.; Barinaga, C. J.; Smith, M. R. J. Anal. Atom. Spectrom. 1994, 9, 1053.

15. Walder, A. J.; Koller, D.; Reed, N. M.; Hutton, R. C.; Freedman, P. A. I. Ansl. Atom. Spectrum. 1993, 8, 1037.

16. Allen, L. A.; Pang, H. M.; Warren, A. R.; Houk, R. S. I. Anal. Atom. Spectrom. 1995, 10, 267.

17. Mattauch, J.; Herzog, R. Z. Phys. 1934, 89, 786.

18. Nier, A. O.; Hayden, J. L. Int. J. Mass Spectrom. Ion Phys. $1971,6,339$.

19. Murphy, D. M.; Mauersherger, K. Rev. Sci. Instrum. 1985, 56 , 220.

20. Murphy, D. M.; Maurersberger, K. Int. J. Mass Spectrom. Ion Proc. 1987, 76, 85.

21. Sinha, M. P.; Giffin, C. E.; Norris, D. D.; Ester, T.; Vilker, V. L.; Friedlander, S. K. Colloid Interface Sci. 1982, 87, 140.

22. Sinha, M. P. In Particles in Gases and Liquids: Detection, Characterization and Control; Mittal, K. L., Ed.; Plenum Press: New York, 1990; p 197.

23. Arrowsmith, P. Anal. Chem. 1987, 59, 1437.

24. Cromwell, E. F.; Arrowsmith, P. Anal. Chem. 1995, 67, 131.

25. Montaser, A.; Ishii, I.; Clifford, R. H.; Sinex, S. A.; Capar, S. G. Anal. Chem. 1989, 61, 2589

26. Giese, C. F. Rev. Sci. Instrum. 1959, 30, 260.

27. Enge, H. A. Rev. Sci. Instrum. 1959, 30, 248.

28. Sintha, M. P.; Tomassian, A. D. Rev. Sci. Instrum. 1991, 62, 2618.

29. Arrowsmith, P.; Hughes, S. K. Appl. Spectrosc. 1988, 42, 1231.

30. Cromwell, E. F.; Caley, C. E. In Proceedings of the 42 nd ASMS Conference on Mass Spectrometry; Chicago, IL, May 29, 1994; $\mathrm{p}$ 847.

31. Cromwell, E. F.; Arrowsmith, P. Appl. Spectrosc. 1995, 49, 1652.

32. Weast, R. C.; Astle, M. J., Eds. CRC Handbook of Chemistry and Physics, 60th ed.; CRC Press: Boca Raton, FL, 1979; p B-258. 